\title{
Actitudes de los estudiantes de administración de empresas hacia la responsabilidad social corporativa y la ética empresarial
}

\section{Attitudes of business administration students towards corporate social responsibility and business ethics}

\author{
Miguel Ángel LÓPEZ NAVARRO; Mercedes SEGARRA CIPRÉS \\ Universitat Jaume I
}

Recibido: Octubre 2011

Aceptado: Diciembre 2011

\section{Resumen}

El reconocimiento creciente de la importancia de la gestión responsable y ética de las organizaciones debería verse reflejado en el campo de la educación, especialmente en los estudios de administración de empresas. A partir de una muestra de estudiantes de esta especialidad, se analizan sus actitudes en dicho campo. Los resultados muestran que los estudiantes, en su concepción de lo que es una empresa bien gestionada, priorizan el enfoque de los "interesados en la compañía". Por otra parte, se aprecian diferencias entre las percepciones relativas al clima ético actual y al deseado. También se observan diferencias en función del género, pero no del curso académico, lo que denota que la educación recibida no incide sobre las percepciones de los alumnos con respecto a la temática abordada.

Palabras clave: Actitudes de estudiantes, educación en administración de empresas, responsabilidad social corporativa, ética.

\begin{abstract}
The responsible management and ethical values should be reflected in the field of education, especially in studies of business administration. From a sample of university business students, this paper examines their attitudes to responsible and ethical behavior in organizations. The results show that students, in their conception of what a well-run company, are close to a stakeholders' perspective. There are also differences between the perceptions of the current and ideal ethical climate. In addition, differences based on gender, but not according to the academic year, are observed which indicates that the education received does not significantly affect the perceptions of students in the topics addressed.
\end{abstract}

Keywords: Student attitudes, business administration education, social corporate responsibility, ethics. 
En los últimos años se ha generado un interés creciente por todas aquellas cuestiones vinculadas con la dimensión social de la actividad empresarial. Los diferentes aspectos que atañen a la ética y la responsabilidad social corporativa (RSC) ocupan un número cada vez mayor de páginas en las publicaciones académicas, así como en las memorias corporativas de las organizaciones.

Las exigencias crecientes en materia de ética y RSC a las que se enfrentan las empresas en el contexto actual responde, de acuerdo con Nieto (2004), a variaciones tales como el aumento de la presión reguladora, la modificación de las pautas de la demanda en los mercados de consumo y la corrección de los criterios de inversión en los mercados financieros. Tales elementos han modificado sustancialmente las condiciones del entorno en el que compiten las empresas, de manera que la adopción de comportamientos socialmente responsables se incorpora a las organizaciones como un elemento más de su estrategia empresarial, como una forma de diferenciación y posicionamiento en el mercado. Paralelamente, existe un consenso acerca de los beneficios de la ética y la RSC sobre la viabilidad de la empresa a largo plazo. Su uso posibilita que la organización dirija una imagen favorable hacia todos los grupos de interés, lo que a su vez contribuye a mejorar su reputación. Como ha sucedido en otros países, esta perspectiva debería suponer un cambio en el campo de la educación en administración de empresas. De hecho, las actitudes de los estudiantes de esta especialidad, en lo que concierne a un adecuado comportamiento a la hora de gestionar con éxito los negocios, se construyen en buena medida durante su etapa de enseñanza universitaria (De la Calle et al., 2007; Lämsä et al., 2008). La formación de actitudes y valores se produce a partir de un proceso de socialización, por el cual las actitudes de las personas, sus valores, motivaciones y comportamientos se transforman para conformar lo que es visto como deseable en un contexto sociocultural determinado (Berger y Luckmann, 1966). En este sentido, las instituciones de enseñanza superior son reconocidas como agentes de socialización de los estudiantes. En el caso de los estudios sobre administración de empresas, las escuelas de negocios y los centros de enseñanza superior especializada en estos temas representan agentes de socialización puesto que establecen los fundamentos básicos para la formación de las actitudes, las habilidades profesionales y la orientación de los valores de las próximas generaciones de profesionales en el mundo de los negocios (Gómez-Mejía, 1983; Lämsä et al., 2003; Lämsä et al., 2008).

Bien es cierto que el interés en el plano educativo por las cuestiones relativas a la ética y la RSC se ha acrecentado en los últimos años, en buena medida como consecuencia de los escándalos empresariales que se han producido en la última década (Jennings, 2004; Luthar y Karri, 2005; Lämsä et al., 2008). Sin embargo, algunos autores, como por ejemplo Ghoshal (2005) o Kashyap et al. (2006), señalan que la educación en las escuelas de negocios es todavía inadecuada para dirigir los cambios en el comportamiento ético de los futuros profesionales de la administración de empresas, dado que los centros de enseñanza todavía priorizan las teorías y los modelos económicos que enfatizan el valor de los accionistas sobre los restantes grupos de interés. En cualquier caso, tratar de analizar en qué medida los estudiantes priorizan alguno de los 
dos modelos contrapuestos relativos a quien debe ostentar el énfasis en la orientación de la compañía -accionistas frente a los grupos interesados en la compañía- constituye una cuestión relevante y escasamente explorada.

A la vista de lo expuesto, los objetivos del presente trabajo residen en aproximar una visión relativa a las actitudes y valores de los estudiantes de administración de empresas de una universidad española en relación con la ética y la RSC. Concretamente, se trata de dar respuesta a las siguientes preguntas: ¿Cuál es su concepción acerca de lo que es una empresa bien gestionada desde el punto de vista de la RSC? ¿Dicha concepción está más próxima a un modelo basado en los intereses del accionista o de los partícipes en la compañía? ¿Cuál es su percepción acerca del clima ético actual y el deseable? ¿Influye el género y el nivel de estudios -la educación recibida- en las cuestiones anteriores?

\section{Actitudes y percepciones de los estudiantes}

La RSC asume implícitamente que las empresas, a través de sus decisiones y actuaciones, manifiestan una preocupación que va más allá de las cuestiones meramente legales (Doh y Guay, 2006; McWilliams y Siegel, 2001; Van Marrewijk, 2003; Boatright, 2003). En dicho concepto subyace la obligación de las empresas de emprender sus actuaciones y asignar sus recursos con el fin último de mejorar el bienestar social, actuando con respecto a los principios éticos y morales de la dignidad humana. De esta manera, las organizaciones que tienen un comportamiento socialmente responsable delimitan sus estrategias y ejecutan procedimientos internos de gestión teniendo en cuenta no sólo la dimensión económica de sus acciones sino también las dimensiones social y ambiental (Nieto, 2004).

Buena parte de la argumentación en torno a la consideración de la RSC en el ámbito de la empresa ha venido acompañada del clásico debate en torno a dos posicionamientos enfrentados: el denominado enfoque de los accionistas frente al de los interesados en la compañía. El primero de ellos, de marcado carácter financiero, sustenta su argumentación en que el desvío de la empresa de sus obligaciones, que no son otras que competir en el mercado para lograr la máxima eficiencia que garantice la creación de valor para los accionistas, acabaría lesionando los legítimos intereses de éstos. Por lo tanto, se asume que no corresponde a la empresa implicarse en actuaciones de índole social, quedando acotadas sus obligaciones al cumplimiento de la legalidad vigente. Las palabras de Milton Friedman, premio nobel de Economía y máximo exponente de esta corriente, expresan con claridad la idea antes apuntada: "El que nuestros dirigentes de empresa aceptaran la idea de que les corresponde una responsabilidad social que no sea el obtener el mayor beneficio posible para sus accionistas sería tanto como socavar los cimientos de nuestra sociedad libre" (Friedman, 1966: 173).

En el otro extremo, el denominado enfoque de los interesados o partícipes, sostiene que el deber de la empresa reside en contribuir al bienestar de la sociedad en un sentido 
amplio, lo que incluye el bienestar de todos y cada uno de los interesados. De esta manera, la capacidad de la empresa para generar dicho bienestar vendrá determinada por su relación con los distintos grupos de interés (Carroll, 1989; Freeman, 1984; 1999; Mitchell et al., 1997; Post et al., 2002). Tal y como señala Ramiro (2009), es este segundo posicionamiento el que se ha ido haciendo predominante en el transcurso del tiempo y, a día de hoy, la mayoría de las grandes corporaciones asumen la conveniencia de internalizar un planteamiento acorde con los objetivos de los distintos interesados, aunque ello responda simplemente a la creencia de que ser una organización responsable es bueno para sus intereses empresariales a largo plazo.

Así, el denominado enfoque de los interesados constituye un poderoso sustrato argumental que sustenta, en esencia, los planteamientos favorables a la RSC. La asunción de dicho enfoque pasa también por la adecuada especificación de quienes deben ser considerados realmente como interesados. Como señala Rodríguez (2002), podemos adoptar un criterio amplio en el que incluir a cualquier individuo, grupo u organización que pueda incidir o pueda verse afectado por las actividades de la empresa, o bien un criterio más estricto que acote el campo a los individuos, grupos u organizaciones identificables y relevantes de los que la empresa depende para su supervivencia. En todo caso, el gran reto de los gestores de la empresa actual reside en reconocer los intereses mutuos entre la empresa y sus interesados. A partir de ahí se establece un proceso de negociación que busca resolver posibles conflictos y equilibrar el reparto de valor entre los diferentes partícipes. Por otra parte, se asume que la adopción de prácticas de RSC va a tener efectos positivos en el largo plazo que incrementarán los beneficios de la empresa y, de esta manera, se restituirá en buena medida la teórica cesión de poder desde los accionistas hacia los otros grupos de interés de la organización.

Además de la cuestión relativa a la orientación de los estudiantes de administración de empresas hacia el enfoque de los accionistas o del de los interesados en la compañía, el presente estudio, tomando como referencia los trabajos de Luthar et al., (1997) y Luthar y Karri (2005), centra también su atención en las percepciones de dichos estudiantes acerca del clima ético actual (percepción de la relación existente entre las prácticas éticas y los resultados de la actuación empresarial) y el clima ético ideal (percepción sobre la relación ideal entre las prácticas éticas y los resultados de la actuación empresarial). Como señalan Luthar y Karri (2005), el análisis de ambas magnitudes es importante en la medida que las percepciones de la relación (actual y deseada) entre las prácticas éticas y los resultados empresariales nos ofrece información acerca de los valores morales de la sociedad y, particularmente, de las aspiraciones morales de los individuos, además de sugerirnos en qué medida los comportamientos de carácter ético por parte de las organizaciones son reconocidos y recompensados.

Por otra parte, diversos trabajos han tratado de analizar la influencia que sobre las actitudes éticas ejercen factores tales como el género o el nivel de estudios. Las posibles diferencias entre hombres y mujeres en lo que concierne a su orientación ética ha sido una cuestión histórica sujeta a discusión. Y si bien la evidencia no es unánime, y el debate continúa en la literatura, la mayoría de los estudios muestran diferencias entre 
hombres y mujeres, siendo estas últimas las que reflejan una orientación ética más acusada (Borkowski y Ugras, 1992; Luthar et al., 1997; Conroy y Emerson, 2004; Luthar y Karri, 2005). Parece, por tanto, que las mujeres son más conscientes de los problemas éticos en el mundo de los negocios, y tienden a vincular en mayor grado la existencia de una estrecha relación entre las prácticas éticas de la organización y sus resultados. La literatura ha tratado de explicar estas diferencias de comportamientos éticos asociadas al género como consecuencia de los procesos de socialización de hombres y mujeres (White y Dooley, 1993). Este dato constituye un elemento relevante a la hora de valorar las actitudes y percepciones que tienen los estudiantes de administración de empresas, futuros directivos y empresarios, especialmente porque los principales puestos directivos de las empresas los ocupan mayoritariamente los hombres. Según el International Business Report de 2011 elaborado por la compañía Grant Thornton, las mujeres en España ocupan el 22\% de los cargos directivos en las empresas, un $2 \%$ más que la media mundial que es del $20 \%$. En comparación con el resto de países del mundo, Tailandia ocupa la posición en el ranking con un $45 \%$ de mujeres con cargos directivos, seguidos por Georgia (40\%), Rusia (36\%), Hong Kong y Filipinas (ambos con un 35\%). En cambio, los países con los menores porcentajes los representan India, Emiratos Árabes y Japón con porcentajes inferiores al 10\%.

Paralelamente, también ha sido analizada en la literatura la relación entre los valores éticos de los estudiantes y su estatus académico. Estudios como el de Borkowski y Ugras (1998) o el de Luthar et al. (1997) muestran la existencia de diferencias entre el comportamiento ético y el nivel o curso en que se encuentran los estudiantes, observándose una mayor orientación ética en el caso de los estudiantes de cursos inferiores. La argumentación que sostienen dichos autores para justificar tales diferencias reside en que los estudiantes de los últimos cursos, bien como consecuencia de su mayor contacto con el mundo laboral, o por su proximidad al mismo, son más utilitaristas (priorizan la maximización de los beneficios y la reducción de los costes en sus aproximaciones a los dilemas éticos) que los estudiantes de los primeros cursos, quienes con menor probabilidad han estado expuestos a los rigores y las presiones de la actividad laboral.

\section{Método de investigación}

El trabajo de campo se desarrolló durante el mes de mayo de 2008 a través de una encuesta presencial dirigida a los estudiantes de $1^{\circ}$ y $4^{\circ}$ curso de la Licenciatura en Administración y Dirección de Empresas de una universidad pública española (Universitat Jaume I). La población objeto de estudio constaba de 404 alumnos, de los cuales participaron en nuestra investigación 174 , lo que constituye un error muestral del $\pm 5,6$ a un nivel de confianza del $95 \%$. El $57 \%$ eran estudiantes de primer curso, mientras que el $43 \%$ restante se encontraban cursando el último año de la titulación. En cuanto a la distribución por sexos, el $63 \%$ eran mujeres y el $37 \%$ hombres. La investigación planteada tiene un marcado carácter exploratorio puesto que se centra en estudiantes pertenecientes a una sola institución. En este sentido nuestra idea inicial era analizar la 
realidad más cercana, al igual que han hecho estudios previos sobre el tema (por ejemplo, Lämsä et al., 2008), si bien parece razonable asumir que las actitudes de dichos alumnos pueden ser similares a las del resto de estudiantes españoles que cursan estudios de Administración y Dirección de Empresas en otras universidades públicas. El cuestionario utilizado está compuesto de dos secciones:

1. En primer lugar, para medir las actitudes de los estudiantes hacia valores de RSC utilizamos las cuestiones desarrolladas por el Instituto Aspen dentro del programa Initiative for Social Innovation through Business (Aspen ISIB). En 1999, el Aspen ISIB realizó un estudio longitudinal midiendo los efectos de la educación de los MBA en las actitudes de los estudiantes sobre las funciones y responsabilidades en los negocios. Dicho estudio fue utilizado en 2001 para la elaboración de un informe titulado "Where will they lead?". Y ese mismo estudio fue replicado en el tiempo, en los años 2002 y 2007. Posteriormente, otras investigaciones en esta línea, como la desarrollada por Lämsä et al., (2008), han utilizado el mismo cuestionario para establecer conclusiones en diferentes entorno geográficos. En nuestro caso, y tomando como referencia dichos estudios, analizamos específicamente las actitudes de los estudiantes sobre una empresa bien gestionada a partir de la evaluación de doce características, haciendo uso de una escala Likert de 5 puntos.

2. En segundo lugar, y en base a la investigación de Luthar et al., (1997), planteamos a los estudiantes que valorasen una serie de cuestiones relativas a dos dimensiones sobre la ética de los negocios. La primera se centraba en evaluar la percepción del clima ético actual ("lo que es"), mientras que la segunda pretendía evaluar la percepción del clima ético ideal ("lo que debería ser"). Tomando como referencia el trabajo antes apuntado, los estudiantes fueron invitados a responder a una batería de preguntas relacionadas con ambas dimensiones, utilizando para ello escalas Likert de 5 puntos.

\section{Resultados}

En la tabla 1 se muestran los resultados de las valoraciones efectuadas por los alumnos acerca de una empresa bien gestionada. Según estos resultados, las acciones que mejoran las condiciones de los clientes y de los empleados fueron las características más destacadas de una empresa bien gestionada, mientras que obtener altos beneficios para los accionistas fue considerada como el rasgo menos relevante para una empresa de estas características. Por tanto, dichos resultados denotan que los estudiantes encuestados están más próximos a la perspectiva de los interesados en la compañía que a un modelo basado en los intereses de los accionistas. 


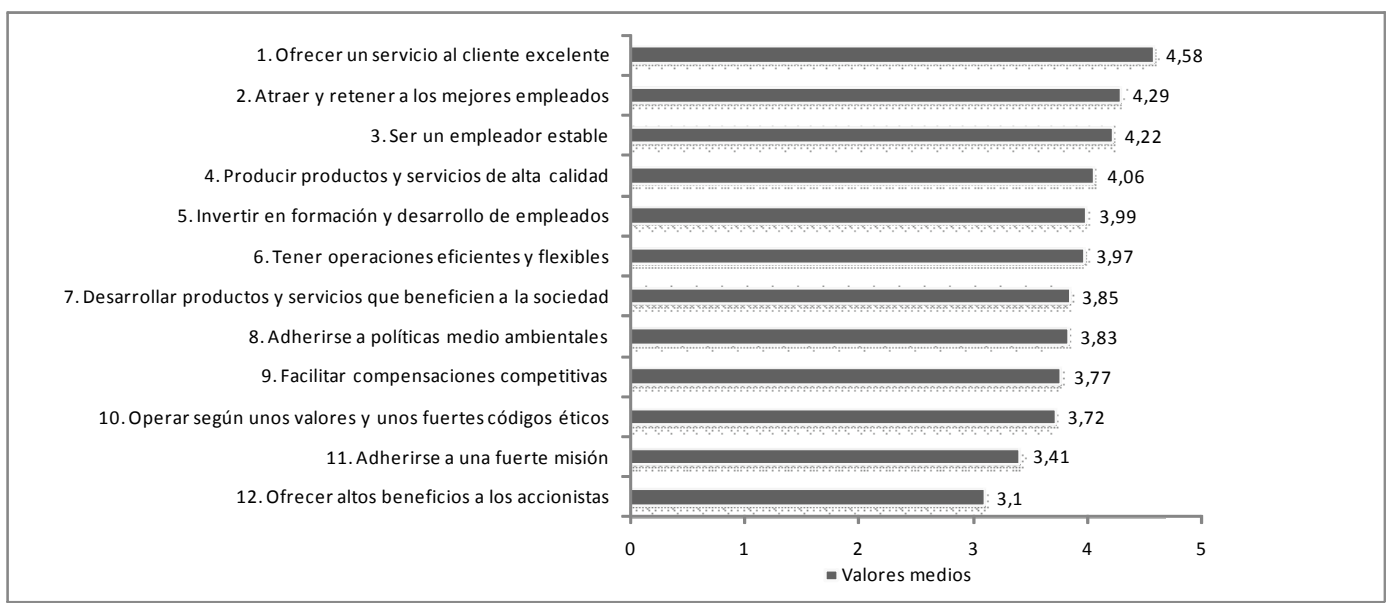

Tabla 1. Concepción de una empresa bien gestionada según los estudiantes universitarios de administración de empresas

A continuación analizamos el efecto que el género y la fase de estudio en la que se encuentra el estudiante tiene sobre sus actitudes hacia los valores de RSC utilizando el análisis ANOVA (con carácter previo a la realización de dichos análisis, en esta y otras partes del trabajo, se comprobó que los datos reunían los requisitos previos para su utilización). Respecto al género de los encuestados, encontramos diferencias significativas en cuatro de las características analizadas (tabla 2). Los resultados muestran que las mujeres, a diferencia de los hombres, valoran las dimensiones sociales y medioambientales como características clave de una empresa bien gestionada. En general, las mujeres, a diferencia de los hombres, dieron un mayor peso en ocho de las doce características analizadas, lo que denota un mayor grado de compromiso hacia la perspectiva de los interesados en la compañía.

\begin{tabular}{|l|c|c|c|c|}
\hline \multicolumn{1}{|c|}{ Empresa bien gestionada } & Género & N & Media & F-test \\
\hline Tener operaciones eficientes y flexibles & Hombre & 63 & 3,79 & $4,953^{* *}$ \\
\cline { 2 - 4 } & Mujer & 106 & 4,06 & \\
\hline Invertir en formación y desarrollo de empleados & Hombre & 63 & 3,79 & $5,256^{* *}$ \\
\cline { 2 - 4 } & Mujer & 106 & 4,07 & \\
\hline \multirow{2}{*}{$\begin{array}{l}\text { Desarrollar productos y servicios que benefician } \\
\text { a la sociedad }\end{array}$} & Hombre & 63 & 3,57 & \multirow{2}{*}{$9,677^{* * *}$} \\
\cline { 2 - 4 } Adherirse a políticas medioambientales & Mujer & 106 & 4,02 & \multirow{2}{*}{$7,263^{* * *}$} \\
\cline { 2 - 4 } & Hombre & 63 & 3,60 & \\
\cline { 2 - 4 } Nota: $* * p<0,05 ; * * * p<0,01$ & Mujer & 106 & 4,00 & \\
\hline
\end{tabular}

Tabla 2. Características de una empresa bien gestionada desde una perspectiva de género 
Respecto a la influencia de la fase de estudios, encontramos diferencias estadísticamente significativas en tres características (tabla 3). Particularmente, los estudiantes que se encontraban en la fase inicial de sus estudios valoraron más las características relacionadas con el entorno en general, es decir, aquellos valores que benefician a la sociedad o que implican la implantación de políticas medioambientales como modelo de empresa bien gestionada. Sin embargo, los estudiantes que se encontraban al final de la fase de estudios valoraron más las acciones que mejoraban las relaciones con empleados en su definición de empresa bien gestionada, puesto que en breve se incorporarán al mercado laboral, en la mayoría de los casos en trabajos por cuenta ajena.

\begin{tabular}{|l|c|c|c|c|}
\hline \multicolumn{1}{|c|}{ Empresa bien gestionada } & $\begin{array}{c}\text { Fase de estu- } \\
\text { dios }\end{array}$ & $\mathbf{N}$ & Media & \multirow{2}{*}{ F-test } \\
\hline \multirow{2}{*}{$\begin{array}{l}\text { Invertir en formación y desarrollo de empleados } \\
\text { (1) }\end{array}$} & Inicial & 100 & 3,88 & \multirow{2}{*}{$5,080^{* *}$} \\
\cline { 2 - 4 } $\begin{array}{l}\text { Desarrollar productos y servicios que benefician } \\
\text { a la sociedad }\end{array}$ & Final & 74 & 4,14 & \\
\cline { 2 - 4 } & Inicial & 100 & 4,02 & \multirow{2}{*}{$7,772^{* * *}$} \\
\hline \multirow{2}{*}{ Adherirse a políticas medioambientales } & Final & 74 & 3,62 & \\
\cline { 2 - 4 } & Inicial & 100 & 4,01 & \multirow{2}{*}{$7,949^{* * *}$} \\
\hline Nota: $* * p<0,05 ; * * * p<0,01$ & Final & 74 & 3,60 & \\
\hline
\end{tabular}

(1) En relación con este análisis, señalar que el resultado de la prueba de Levene no permitía asumir el supuesto de homogeneidad de varianzas. A este respecto, se procedió a efectuar un contraste de medias a través de la $t$ de Student entre los dos grupos considerados (dicho análisis nos ofrece resultados en los supuestos de igualdad o no de varianzas) $\mathrm{y}$, asumiendo varianzas desiguales, el valor del estadístico $t$ era de 2,359 y el valor "p" asociado de 0,19 , lo que nos permite afirmar que existen diferencias entre ambos grupos al nivel de significación 0,05.

Tabla 3. Características de una empresa bien gestionada según la fase de estudios en la que se encuentran los estudiantes

La siguiente etapa de nuestro análisis radica en la evaluación de las opiniones de los estudiantes sobre el clima ético existente y el ideal. En las tablas 4 y 5 se exponen los resultados de dicho análisis. La conclusión más visible, a raíz de la evaluación comparativa entre los ítems coincidentes en ambas tablas, reside en la existencia de diferencias globales claras entre el clima ético existente y el clima ético deseado. De alguna manera se constata que la percepción de la relación actual entre las prácticas éticas y los resultados de la actividad empresarial es inferior a la percepción de lo que dicha relación debería ser en una situación ideal o deseable. En consecuencia, ello indicaría un cierto rechazo, por parte de los estudiantes de administración de empresas encuestados, a los valores morales de la sociedad actual y a cómo el mercado premia o sanciona los buenos comportamientos de la empresa en el campo de la ética. 


\begin{tabular}{|l|c|}
\hline \multicolumn{1}{|c|}{ Percepción de "lo que es" } & Media \\
\hline La buena ética es un buen negocio & 3,16 \\
\hline Cuando una empresa no actúa éticamente, será sancionada reduciendo sus beneficios & 2,82 \\
\hline Es necesario ser ético para ser un directivo de éxito & 2,96 \\
\hline $\begin{array}{l}\text { El éxito del libre mercado y las instituciones democráticas depende de que los directi- } \\
\text { vos actúen éticamente }\end{array}$ & 3,08 \\
\hline Una empresa necesita ser ética para ser admirada & 3,26 \\
\hline
\end{tabular}

Tabla 4. Clima ético actual ("lo que es")

\begin{tabular}{|l|c|}
\hline \multicolumn{1}{|c|}{ Percepción de "lo que debería ser” } & Media \\
\hline La buena ética es un buen negocio & 3,80 \\
\hline Cuando una empresa no actúa éticamente, será sancionada reduciendo sus beneficios & 3,85 \\
\hline Es necesario ser ético para ser un directivo de éxito & 3,77 \\
\hline $\begin{array}{l}\text { El éxito del libre mercado y las instituciones democráticas depende de que los directi- } \\
\text { vos actúen éticamente }\end{array}$ & 3,59 \\
\hline Una empresa necesita ser ética para ser admirada & 3,88 \\
\hline
\end{tabular}

Tabla 5. Clima ético ideal (“lo que debería ser”)

Por otra parte, y para cada una de las dos dimensiones -clima ético actual e ideal-, se procedió a analizar la existencia de posibles diferencias significativas en función del género y del nivel de estudios. En la tabla 6 se muestran los resultados relativos al clima ético existente en función del género, observándose diferencias significativas en la mayor parte de los ítems evaluados, reflejando una mayor orientación ética en el caso de las mujeres. En lo que concierne a las diferencias de género con respecto al clima ético ideal (tabla 7), los ítems donde se dan tales diferencias muestran también valores más elevados en la dirección antes apuntada. Todo ello permite constatar una mayor sensibilidad de las mujeres con respecto a los valores éticos en el campo de los negocios. En lo que concierne a la etapa de los estudios, los análisis efectuados han revelado que no existen diferencias significativas entre los alumnos de primero y de cuarto en lo relativo al clima ético actual e ideal.

\begin{tabular}{|c|c|c|c|}
\hline Percepción de "lo que es" & Género & Media & F-test \\
\hline \multirow[t]{2}{*}{ La buena ética es un buen negocio } & Hombre & 2,84 & \multirow{2}{*}{$11,707 * * *$} \\
\hline & Mujer & 3,35 & \\
\hline \multirow{2}{*}{$\begin{array}{l}\text { Cuando una empresa no actúa éticamente, será sancionada } \\
\text { reduciendo sus beneficios }\end{array}$} & Hombre & 2,49 & \multirow[b]{2}{*}{$8,522 * * *$} \\
\hline & Mujer & 3,02 & \\
\hline \multirow[t]{2}{*}{ Es necesario ser ético para ser un directivo de éxito } & Hombre & 2,63 & \multirow{2}{*}{$9,438 * * *$} \\
\hline & Mujer & 3,15 & \\
\hline \multirow{2}{*}{$\begin{array}{l}\text { El éxito del libre mercado y las instituciones democráticas } \\
\text { depende de que los directivos actúen éticamente }\end{array}$} & Hombre & 2,80 & \multirow[b]{2}{*}{$6,910 * * *$} \\
\hline & Mujer & 3,24 & \\
\hline
\end{tabular}

Tabla 6. Clima ético actual ("lo que es") desde una perspectiva de género 


\begin{tabular}{|l|l|c|c|}
\hline \multicolumn{1}{|c|}{ Percepción de "lo que debería ser" } & Género & Media & F-test \\
\hline La buena ética es un buen negocio & Hombre & 3,44 & \multirow{2}{*}{$14,388^{* * *}$} \\
\cline { 2 - 3 } & Mujer & 4,00 & \\
\hline $\begin{array}{l}\text { El éxito del libre mercado y las instituciones democráticas } \\
\text { depende de que los directivos actúen éticamente }\end{array}$ & Hombre & 3,38 & \multirow{2}{*}{$4,746 * *$} \\
\cline { 2 - 3 } Nota: $* * p<0,05 ; * * * p<0,01$ & Mujer & 3,72 & \\
\hline
\end{tabular}

Tabla 7. Clima ético ideal ("lo que debería ser") desde una perspectiva de género

\section{Discusión}

Los resultados de esta investigación sugieren que los estudiantes de administración de empresas encuestados definen a una empresa bien gestionada desde la perspectiva de los interesados en la compañía, haciendo especial hincapié en el compromiso con los empleados y los clientes. En general, los resultados alcanzados son muy similares a los obtenidos recientemente por dos estudios sobre el mismo tema con estudiantes finlandeses (Lämsä et al., 2008) y estadounidenses (Aspen Institute, 2008), aunque con algunas diferencias significativas. Por una parte, nuestros resultados muestran que las valoraciones efectuadas por los estudiantes encuestados están más cercanas a las de los estudiantes finlandeses que a las de los americanos. Así, por ejemplo, al igual que los estudiantes finlandeses, valoran la obtención de altos beneficios para los accionistas como la característica menos importante, mientras que los estudiantes americanos la sitúan en el noveno lugar sobre doce posibles. En cambio, una diferencia notable se refiere a la importancia de la estabilidad laboral para los estudiantes encuestados, dado que estos la consideran como una de las principales características de una empresa bien gestionada, mientras que los entrevistados estadounidenses la consideran como la menos relevante.

Por lo que respecta a las diferencias en las percepciones de los estudiantes sobre cómo es el clima ético en la actualidad y sobre cómo debería ser, los resultados indican que los encuestados perciben la relación entre las prácticas éticas y los resultados empresariales bastante alejada de sus expectativas sobre una situación ideal. En lo que concierne a las diferencias en las actitudes de los estudiantes sobre la RSC y el clima ético en función del género, los resultados de nuestra investigación estarían en consonancia con la literatura dominante (Borkowski y Ugras, 1992; Luthar et al., 1997; Conroy y Emerson, 2004; Luthar y Karri, 2005), constatándose que las mujeres tienen una actitud más favorable hacia los comportamientos éticos y su relación con los resultados empresariales y, además, valoran de forma más positiva las dimensiones medioambiental y social de una empresa como rasgos característicos de una organización bien gestionada. En lo relativo al estatus académico, si bien se observa una actitud más favorable hacia la relación entre empresa y empleados en los alumnos de cuarto curso, que 
sin duda puede venir explicada por la mayor cercanía a su incorporación al mercado laboral, por otra parte se constata la ausencia de diferencias en lo que respecta a su percepción del clima ético actual e ideal. Esto último no estaría en consonancia con los resultados de trabajos como los de Borkowski y Ugras (1992) o Luthar et al. (1997), donde se apunta una menor orientación ética de los estudiantes de cursos superiores. El mayor utilitarismo en el plano ético de estos estudiantes es justificado por dichos autores a partir del mayor contacto que han tenido con el mundo laboral y, por tanto, por haber estado expuestos en mayor medida a los rigores y las presiones de la actividad laboral. Sin embargo, y a diferencia de lo que ocurre en la cultura estadounidense, en el caso español es relativamente bajo el número de alumnos que compatibilizan sus estudios universitarios con la actividad laboral, circunstancia que puede justificar en buena medida la ausencia de diferencias.

\section{A modo de conclusión}

Como hemos podido observar no existen grandes desviaciones entre las actitudes que los alumnos de cursos iniciales y finales tienen respecto a los valores de RSC y ética empresarial. Este resultado nos puede llevar a pensar en qué medida, en la actualidad, la educación superior recoge en sus planes de estudio la importancia que la sociedad otorga al papel de la RSC y a la ética empresarial. Según el estudio "Una aproximación al estado de la RSE en la enseñanza universitaria" de 2010, elaborado por el Club de Excelencia en Sostenibilidad y el Ministerio de Trabajo, la RSC sigue siendo una materia con escasa presencia en la enseñanza universitaria. Según el citado estudio, no existe ningún grado específico sobre RSC y únicamente en cinco universidades se ha introducido una asignatura obligatoria de RSC en sus grados de ámbito empresarial. Fuera de estos, la RSC está escasamente representada y únicamente aparece como asignatura optativa en grados como Ciencias ambientales, Estudios ingleses o Enfermería. En cambio, tal y como se señala en el dicho estudio, esta materia está recibiendo una mayor atención a través de estudios de postgrado especializados, lo que reflejaría una cierta demanda de la sociedad hacia estas cuestiones.

Para finalizar, señalar que si bien la formación ética debe estar presente en cualquier ámbito de la educación universitaria (Agejas et al., 2007), no es menos cierto que los aspectos ligados a la ética y la responsabilidad social cobran una mayor relevancia en el caso de las titulaciones de ámbito económico y empresarial, habida cuenta que tales titulaciones se dirigen a la formación de profesionales liberales o gestores que van a dirigir las empresas, constituyéndose éstas en la piedra angular del modelo económico que sustenta nuestra sociedad. Y en el análisis de dicha cuestión se inscribe el presente trabajo. Pero más allá de éste, sería interesante que en futuros estudios se profundizase en el análisis del impacto que la formación en valores representa en los nuevos estudios de grado en materia económica y empresarial ampliando la muestra a un mayor número de centros de educación superior. 


\section{Referencias bibliográficas}

AGEJAS, J. A., PARADA, J. L. Y OLIVER, I. (2007). La enseñanza de la ética profesional en los estudios universitarios. Revista Complutense de Educación, 18 (2), $67-$ 84.

ASPEN INSTITUTE (2008). Where will the lead? MBA student attitudes about business \& society. The Aspen Institute, Center for Business Education. New York.

BERGER, P. I. Y LUCKMANN, T. (1966). The social construction of reality. Doubleday: New York.

BOATRIGHT, J. R. (2003). Ethics and the conduct of business. Prentice Hall: Upper Saddle River, NJ.

BORKOWSKI, S. C. Y UGRAS, Y. J. (1992). The ethical attitudes of students as a function of age sex, and experience. Journal of Business Ethics, 11, 63-69.

- (1998): Business students and ethics: A meta-analysis. Journal of Business Ethics, $17,1117-1127$.

CARROLL, A. B. (1989). Business and society: Ethics and stakeholders management. South-Western: Cincinnati, $\mathrm{OH}$.

CONROY, S. Y EMERSON, T. (2004). Business education and religion: Religiosity as a predictor of ethical awareness among students. Journal of Business Ethics, 50, 383-396.

DE LA CALLE MALDONADO DE GUEVARA, GARCÍA RAMOS, J.M. Y GIMÉNEZ ARMENTIA, P. (2007). La formación de la responsabilidad social en la universidad. Revista Complutense de Educación, 18, 47-66.

DOH, J. P. Y GUAY, T. R. (2006). Corporate social responsibility, public policy and NGO activism in Europe and the United States: an institutional-stakeholder perspective. Journal of Management Studies, 43, 47-73.

FREEMAN, R. E. (1984). Strategic Management: A stakeholder approach. Pitman: Boston, MA.

- (1999). Response divergent stakeholder theory. Academy of Management Review 24, 233-236.

FRIEDMAN, M. (1966). Capitalismo y libertad. Ediciones Rialp: Madrid.

GHOSHAL, S. (2005). Bad management theories are destroying good management practices. Academy of Management Learning \& Education, 4, 75-91.

GÓMEZ-MEJÍA, L.R. (1983). Sex differences during occupational socialization. Academy of Management Journal, 26, 492-499. 
JENNINGS, M. (2004). Incorporating ethics and professionalism into accounting education and research: A discussion of the voids and advocacy for training in seminal works in business ethics. Issues in Accounting Education, 19, 7-26.

KASHYAP, R., MIR, R. Y IYER, E. (2006). Toward responsive pedagogy: Linking social responsibility to firm performance issues in the classroom. Academy of Management Learning \& Education, 5, 366-376.

LÄMSÄ, A., TURJANMAA, P., SÄKKINEN, A. (2003). The values of business education and working life from a gender viewpoint. En Natale S.M., Libertella, A.F. (eds.), Business Education and Training: A value laden process (91-102). Instructed by reason, University Press of America, Lanham.

LÄMSÄ, A., VEHKAPERÄ, M., PUTTONEN, T. Y PESONEN, H. (2008). Effects of business education on women and men students' attitudes on corporate responsibility in society. Journal of Business Ethics, 82, 45-58.

LUTHAR, H. K., DIBATTISTA, R. A. Y GAUTSCHI, T. (1997). Perception of what the ethical climate is and what is should be: The role of gender, academic status and ethical education. Journal of Business Ethics, 16, 205-217.

LUTHAR, H. K. Y KARRI, R. (2005). Exposure to ethics education and the perception of linkage between organizational ethical behavior and business outcomes. Journal of Business Ethics, 61, 353-368.

MCWILLIAMS, A. Y SIEGEL, D. (2001). Corporate social responsibility: a theory of the firm perspective. Academy of Management Review, 26, 117-127.

MITCHELL, R. K., AGLE, B. R. Y WOOD, D. J. (1997). Toward a theory of stakeholder identification and salience: defining the principle of who and what really counts. Academy of Management Review, 22, 853-856.

NIETO, M. (2004). Responsabilidad social corporative: la última innovación en management. Universia Business Review, primer trimester, 28-39.

POST, J. E., PRESTON, L. E Y SACHS, S. (2002). Managing the extended enterprise: the new stakeholder view. California Management Review, 45, 6-28.

RAMIRO, P. (2009). Las multinacionales y la responsabilidad social corporativa: de la ética a la rentabilidad. En J. Hernández y P. Ramiro (Eds.). El negocio de la responsabilidad: Crítica de la responsabilidad social corporativa de las empresas trasnacionales. Icaria Editorial: Barcelona.

RODRÍGUEZ, J. M. (2002). Teoría de los partícipes y ciudadanía empresarial: una perspectiva pluralista del gobierno de las compañías. Ekonomiaz, 50, 74-109.

VAN MARREWIJK, M. (2003). Concepts and definitions of CSR and corporate sustainability: between agency and communication. Journal of Business Ethics, 44, 95105. 
WHITE, C.S. Y DOOLEY, R.S. (1993). Ethical or practical: An empirical study of students' choices in simulated business scenarios. Journal of Business Ethics, 12, 643-651.

Correspondencia con los autores

Miguel Ángel López Navarro, Mercedes Segarra Ciprés

Departamento de Administración de Empresas y Marketing

Universitat Jaume I

Av. Sos Baynat, $\mathrm{s} / \mathrm{n}$

12071 Castellón de la Plana (España)

e-mail:mlopez@emp.uji.es

e-mail:msegarra@emp.uji.es 